\title{
Superfluid Interface Mixes Classical and Quantum Behavior
}

\author{
Animations illustrate various behaviors of the interface between two \\ oppositely moving superfluids.
}

\section{By David Ehrenstein}

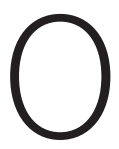

cean waves and swirling patterns on Jupiter are both results of a two-fluid mixing phenomenon called the Kelvin-Helmholtz instability (KHI). A team of theorists has now performed simulations of the $\mathrm{KHI}$ in superfluids and discovered several different behaviors that show a crossover

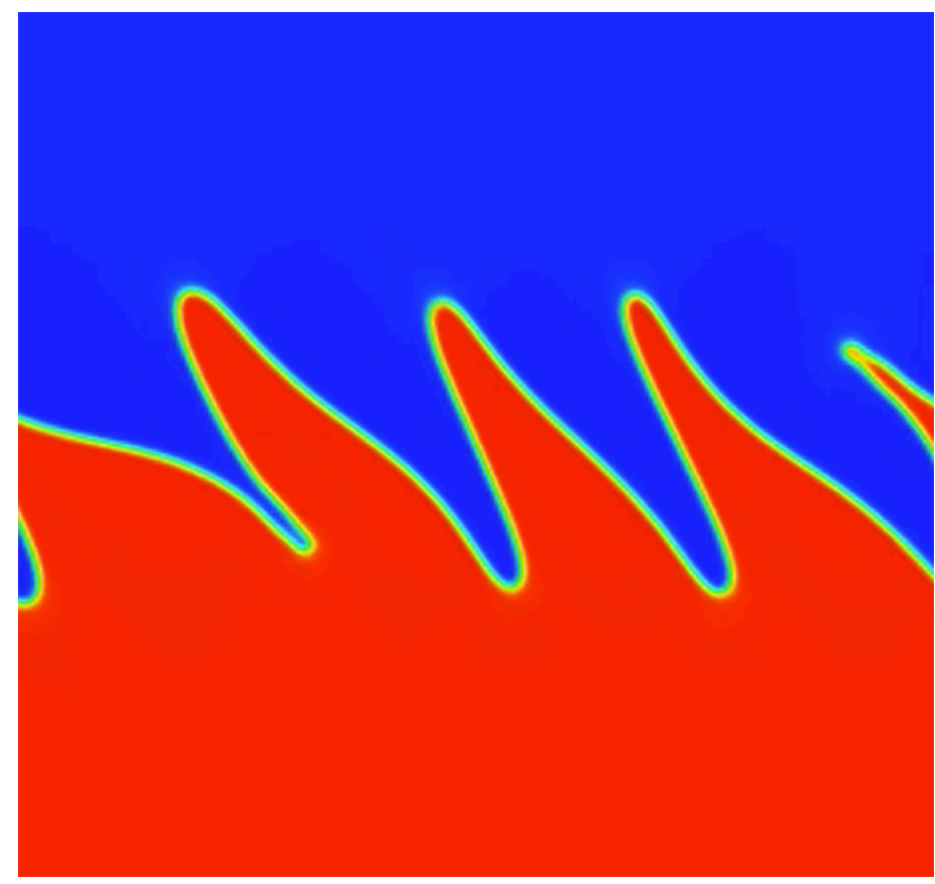

In this simulation, the blue superfluid flows to the left, and the red one flows to the right. With high repulsion between the two and a low relative velocity, the interface is narrow, and the flutter-finger pattern forms, where the fingers tilt like grass blown by the wind. (Animations of other interface patterns are below.)

Credit: H. Kokubo et al. [1]

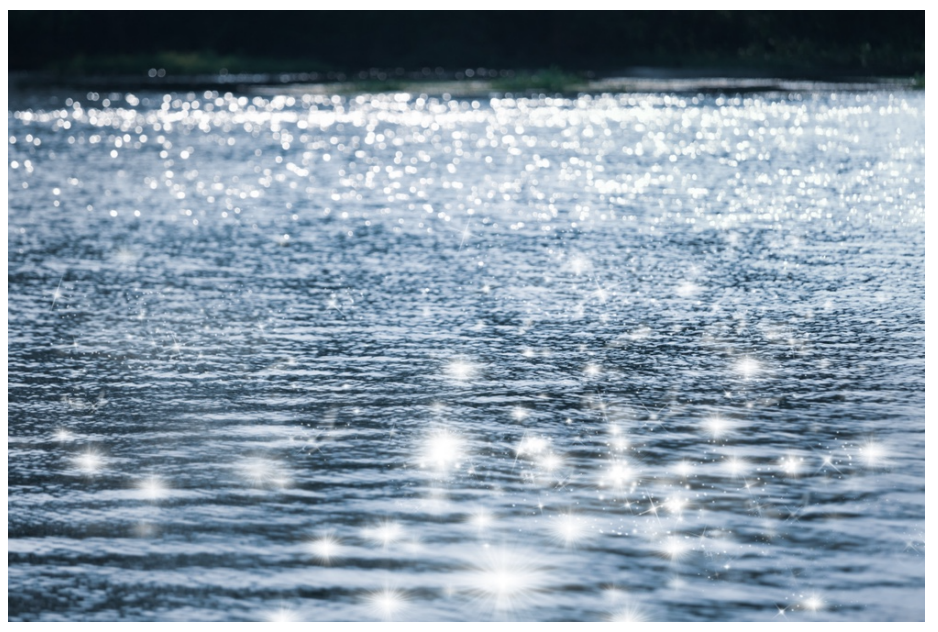

Making waves. Wind blowing across a water surface generates waves through the Kelvin-Helmholtz instability.

Credit: chokchaipoo/stock.adobe.com

from classical to quantum effects [1]. The results cover a wider range of parameters than previous simulations and thus provide the most complete picture to date of the $\mathrm{KHI}$ in superfluids.

In the classical KHI, two fluids share an initially flat boundary and flow at different speeds parallel to the interface, causing the boundary to become wavy or even turbulent. For example, wind can stir up waves in initially flat water. Graduate student Haruya Kokubo of Kindai University, Japan, and his colleagues simulated the interaction of two Bose-Einstein condensates (BECs) - cold atomic gases that lack viscosity and are thus superfluids.

In their simulations, the BECs flowed at the same speed in opposite directions. The team observed various behaviors, 
depending on the fluids' relative speed and on the strength of the repulsion between atoms of the two fluids. The repulsion strength determined the miscibility of the fluids and the thickness of the interface region, where the two species mixed.

With the strongest repulsion and the lowest relative speed, the sharp interface formed what Kokubo and his colleagues call the "flutter-finger" pattern: the waves grow and tilt in response to the relative flow, as "grass flutters in the wind," they write. This behavior is mostly classical.

The team showed that quantum fluid behavior appeared when the interface thickness was similar to the wavelength of the dominant ripples at the fluid boundary. This quantum onset occurred in two situations: The first was when the relative speed was increased, producing a "zipper" pattern, where the tip of each finger breaks off at a certain length, moves sideways, and then re-joins the neighboring finger. The second situation was when repulsion was reduced, causing the interface to thicken and fingers to tilt in the opposite direction from the flutter-finger pattern. The researchers call the pattern "sealskin" in analogy with ski materials whose fine fibers also tilt in a direction against the flow when grabbing onto snow.

David Ehrenstein is a Senior Editor for Physics.

\section{REFERENCES}

1. H. Kokubo et al., "Pattern formation of quantum Kelvin-Helmholtz instability in binary superfluids," Phys. Rev. A 104, 023312 (2021). 


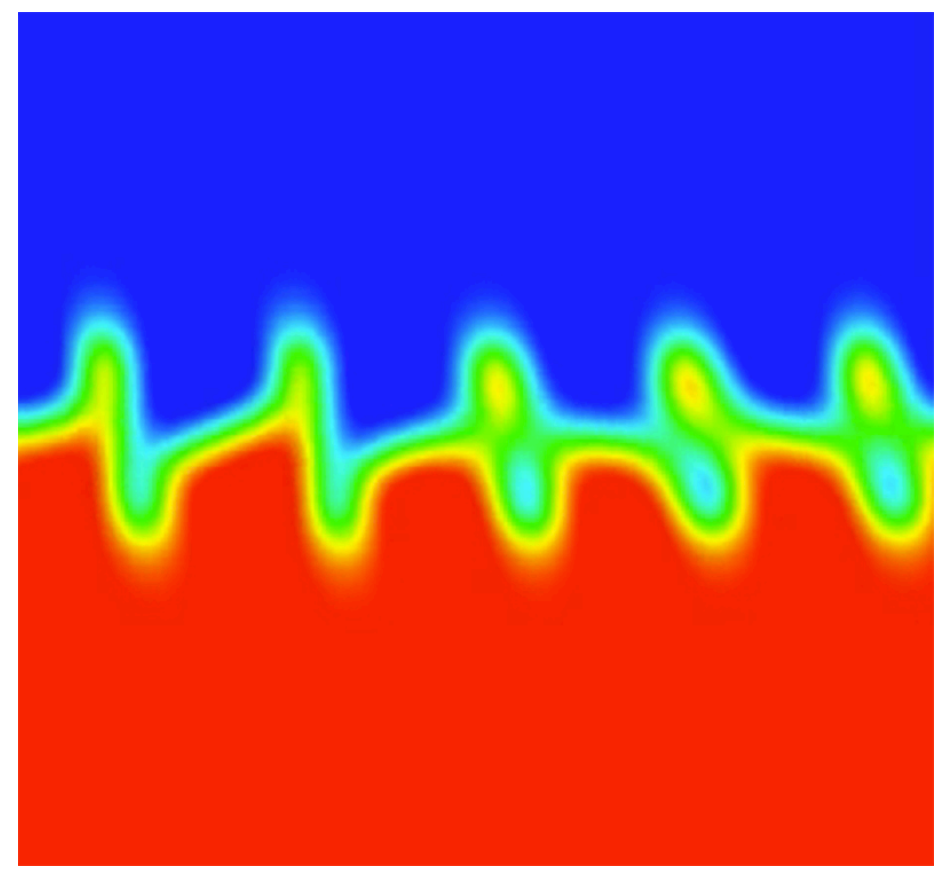

This simulation is similar to the first but with higher relative velocity, leading to ripples with smaller wavelengths (relative to the interface width). The zipper pattern forms, where each finger tip breaks off and then reconnects to a neighboring finger.

Credit: H. Kokubo et al. [1] 


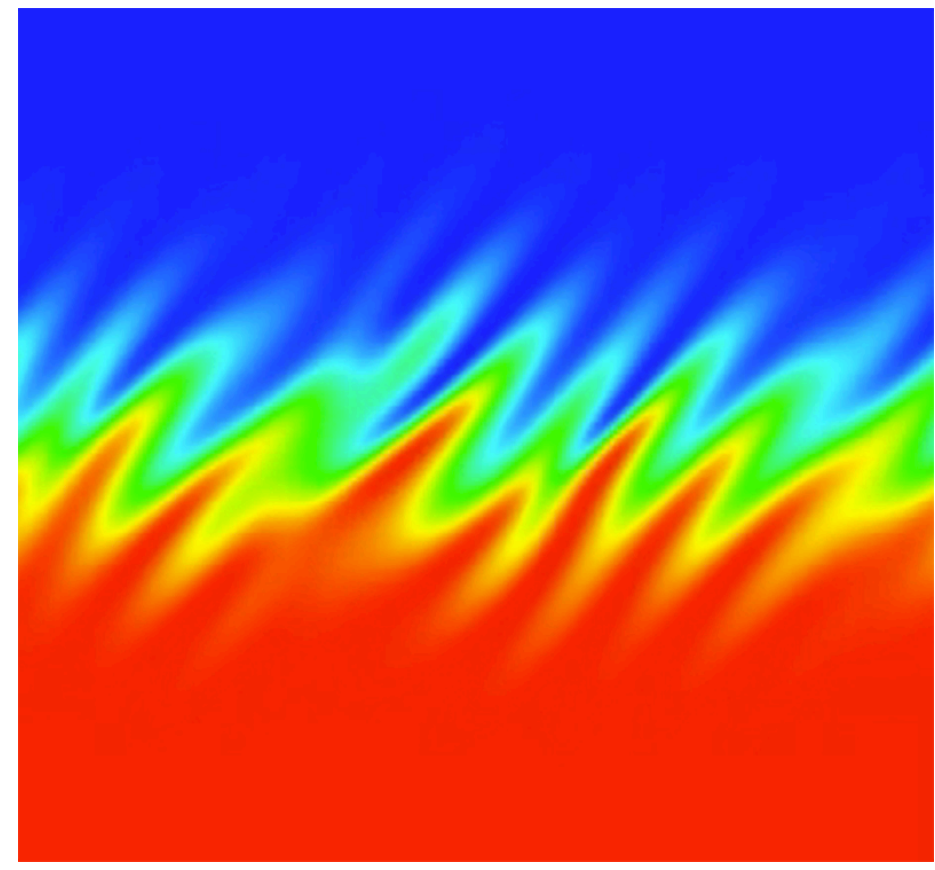

This simulation is similar to the first but with lower repulsion between the two fluids. The resulting interface is wide, and the sealskin pattern forms, where the fingers point into the flow. Credit: H. Kokubo et al. [1] 